\title{
Lifestyle and Disease Prevention
}

National Cancer Institute

\section{Source}

National Cancer Institute. Lifestyle and Disease Prevention. NCI Thesaurus. Code C15898.

Major category of Complementary and Alternative Medicine. This category deals with theories and practices designed to prevent the development of illness, identify and treat risk factors, or support of healing and recovery processes. It is concerned with integrated approaches for the prevention and management of chronic disease-in-general (DIG) or the common determinants of chronic disease. 